\title{
Erros e dificuldades de aprendizagem de estudantes do ensino médio na interpretação da reação química como um sistema complexo
}

\section{Errors and difficulties of high school students' learning in the interpretation of the chemical reaction as a complex system}

Fábia Maria Gomes de Meneses ${ }^{1}$. Isauro Beltrán Nuñez ${ }^{2}$

\begin{abstract}
Resumo: Conhecer a reação química como um sistema complexo é considerado um dos objetivos da educação química no Ensino Médio. O presente estudo objetiva avaliar e compreender esse conhecimento, considerando a resposta de 126 estudantes que responderam uma questão na prova de Química do Vestibular da UFRN, no ano de 2013. Para esse propósito, foram analisadas as respostas e identificados os erros assim como os níveis de desenvolvimento da habilidade de interpretar uma reação química como um sistema complexo, os quais foram relacionados a dificuldades de aprendizagem. Os resultados evidenciam o baixo desempenho dos estudantes e as limitações na compreensão e integração de parâmetros que caracterizam uma reação química. Esses erros podem estar também associados à forma fragmentada de organizar e ensinar os conteúdos relativos às reações químicas gerando dificuldades de aprendizagem, o que exige de novas propostas metodológicas de natureza intradisciplinar.
\end{abstract}

Palavras-chave: Ensino de química. Ensino Médio. Reação química. Sistema complexo. Ensino e aprendizagem.

\begin{abstract}
Knowing the chemical reaction as a complex system is considered one of the aims of high school chemical education. This study aims to evaluate and understand this knowledge, considering the response given by 126 students who answered one question on Chemistry Federal University entrance exam in 2013. For this purpose, the answers were analyzed and the errors identified as well as the levels of ability development in interpreting a chemical reaction as a complex system, and these were related to learning difficulties. The results have shown the low performance of the students and the limitations of the understanding and integration of parameters that characterize a chemical reaction. These errors may also be associated with the fragmented way of organizing and teaching content related to chemical reactions, leading to learning difficulties, which requires new methodological proposals of an intradisciplinary nature.
\end{abstract}

Keywords: Chemistry teaching. High school. Chemical reaction. Complex system. Conceptual mistakes. Teaching and learning.

\footnotetext{
${ }^{1}$ Instituto Federal de Educação, Ciência e Tecnologia do Rio Grande do Norte (IFRN), Parnamirim, RN, Brasil. E-mail: < fabia.meneses@ifrn.edu.br>.

${ }^{2}$ Universidade Federal do Rio Grande do Norte (UFRN), Centro de Ciências Sociais Aplicadas, Natal, RN, Brasil. Orcid: < https://orcid.org/0000-0003-3224-4694>.
} 


\section{Introdução}

O conhecimento da Química tem uma grande importância para a economia mundial, uma vez que, por meio desse conhecimento, é possível compreender aspectos relacionados à cidadania, como os impactos sociais, ambientais e econômicos causados pelos avanços industriais e tecnológicos presentes nas últimas décadas.

Além disso, o estudo das reações químicas tem sido considerado fundamental por pesquisadores e professores, no campo da disciplina escolar Química, e, para compreendê-lo, é necessário conhecer outros conceitos. Estes precisam ser bem entendidos para que não surjam concepções errôneas acerca dessas reações.

Essa importância decorre do fato de as transformações químicas serem consideradas um dos eixos estruturantes dessa disciplina no Ensino Médio, uma vez que ela propicia aos estudantes desenvolver as habilidades necessárias para que compreendam mudanças químicas que ocorrem na natureza física. Portanto, por considerar o conteúdo de reação química um dos principais pilares para compreensão do modo como se organiza e constrói o conhecimento nessa área, torna-se importante investigar quais erros e dificuldades de aprendizagem que se interpõem ao processo de compreensão do mencionado conteúdo.

Nas Diretrizes Curriculares para o Ensino Médio (BRASIL, 2013), no que diz respeito à reação química como objeto de estudo da Química, na matriz das habilidades, ela se apresenta como sistema ao considerar as seguintes dimensões: caracterização das transformações; apectos energéticos e dinâmicos e os modelos de constituição das reações químicas.

Autores como Carbonell e Furió-Más (1987); Furió e Furió (2000); Johnstone (1993, 2000, 2006); Lopes (1992); Mortimer e Miranda (1995); Nardin, Salgado e Del Pino (2005); Rosa e Schnetzler (1998); Talanquer (2006); Uehara (2005) explicitam dificuldades que os estudantes apresentam ao interpretar uma reação química quando observam, de forma separada, parâmetros quantitativos, termodinâmicos, cinéticos, e do equilíbrio. Não obstante, poucas pesquisas têm se dedicado a estudar como os estudantes compreendem as reações químicas como sistemas complexos, nas quais se integram essas dimensões.

Uma das causas associadas aos erros dos estudantes na compreensão de uma reação química como sistema complexo está relacionada ao modo fragmentado e descontextualizado de se ensinar os conteúdos de química, sem estabelecer uma relação entre os próprios conteúdos da formação do conceito como também entre outros conteúdos e saberes que não estão relacionados com as vivências do dia a dia dos alunos, promovendo, assim, o ensino de uma ciência pouco significativa e, na maioria das vezes, difícil de ser realmente compreendida e aplicada pelos alunos.

Segundo Mol e Silva (1996), o ensino da Química possui muitos problemas, dentre eles a simples transmissão de conhecimentos, os conteúdos fragmentados e a falta de motivação para estudar. Estes fazem com que os estudantes tenham ainda mais dificuldades em relacionar os conteúdos entre si. Consideramos a fragmentação como um dos mais importantes problemas e o principal responsável pelas dificuldades de aprendizagem.

Raviolo et al. (2000) também se preocuparam com a problemática da fragmentação dos conteúdos e os consequentes problemas. Há uma fragmentação acentuada dos conteúdos que, por sua vez, não estão relacionados entre si, nem estão dispostos em uma ordem crescente de complexibilidade. Os autores afirmam que esses obstáculos interferem na aprendizagem dos 
conceitos químicos, que devem estar estruturados na disciplina de forma hierarquica e lógica. Pozo et al. (1991), assim como Raviolo et al. (2000) chamaram a atenção para a escassa organização hierárquica do conhecimento conceitual que os alunos têm, dificultando, dessa forma, o estabelecimento de relações entre os conceitos.

As pesquisas publicadas na literatura sobre as dificuldades e os erros relativos à compreensão de uma reação química confirmam a situação de que dimensões importantes na aprendizagem desse conteúdo, como os quantitativos-qualitativos, energéticos, cinéticos e de equilíbrio químico, são apresentados aos estudantes de forma fragmentada.

Nesse sentido, torna-se relevante não só explicitar os erros como também explicitar as causas que podem ser atribuídas a estes em termos de dificuldades de aprendizagem. Ao fazê-lo, é possível ajudar os estudantes a superar as dificuldades de aprendizagem relacionadas ao tema reações químicas, para o qual tem contribuído os estudos de Beltrán Núñez e Uehara (2012), Caamaño (2003, 2007), Meneses (2015), e Pozo e Gómez (2009).

$\mathrm{Na}$ Química, as dificuldades intrínsecas de aprendizagem podem ser explicadas pela existência de três níveis de descrição da matéria. Como referenciado anteriormente, os níveis para Caamaño (2007) são: macroscópico (observacional), microscópico (atômico-molecular) e o representacional (símbolos, fórmulas e equações). Ele afirma que os estudantes devem se movimentar entre esses níveis mediante o uso da linguagem, que não se diferencia de forma explícita nesses níveis.

Dentre as dificuldades intrínsecas da disciplina, é possível identificar o uso de diferentes modelos e teorias utilizados na aprendizagem escolar, pois há necessidade de se utilizarem modelos sofisticados de diferentes teorias para mesma área conceitual dentro do estudo da Química. Esses modelos e teorias exigem a realização de sucessivos processos de integração e diferenciação conceitual ao longo da aprendizagem escolar.

Uma proposta para superar a visão fragmentada dos conteúdos da Química, dentre eles o das reações químicas, tem sido desenvolvida por Beltrán Núñez (1994, 2009), Reshetova (1988), Sálmina (1988) e denominada enfoque sistêmico funcional estrutural.

Para Reshetova (1988), as ideias sistêmicas dialéticas se convertem em aspectos importantes das teorias científicas e da concepção contemporânea do mundo. O tipo sistêmico dialético do pensamento científico não é somente para solucionar os problemas mais complexos de uma atividade transformadora. A solução exitosa e criativa, responsável por uma variedade de problemas, depende da capacidade de pensar de forma sistêmica e dialética como uma necessidade atual da sociedade do conhecimento e da prática humana. Compreender uma reação química como sistema supõe uma visão de sistema de totalidade, como tem apontado Reshetova (1988); Sálmina (1988) e Beltrán Núñez (1994, 2009).

Nesse enfoque, segundo Beltrán Núñez (2009), o objeto de estudo se apresenta na sua totalidade, e se destaca nele a compreensão e a estrutura, que garantem seu comportamento. O tipo de ligação principal, formador do sistema, é a funcional estrutural. O objeto complexo é interpretado como uma formação integrada, na qual as propriedades integradas específicas não se reduzem às propriedades de seus componentes. O objeto de estudo é considerado um conjunto se subsistemas estruturais e funcionais hierarquizados em níveis sistematizados. Dessa forma, a reação química como um sistema complexo supõe a integração das diferentes dimensões (qualitativa, quantitativa, energética, cinética, do equilíbrio químico) que permitem explicar uma reação química, sendo essas diferentes dimensões os subsistemas do sistema complexo. 
Considerando essa problemática, o trabalho tem como finalidade discutir a compreensão de estudantes do Ensino Médio em uma questão discursiva na prova de Química do Vestibular 2013 da Universidade do Rio Grande do Norte (UFRN) acerca das reações química como sistema complexo. Para essa finalidade, foram definidas as seguintes questões de estudo: quais erros e dificuldades de aprendizagem apresentam os estudantes em relação à compreensão dos significados dos termos $\Delta \mathrm{G}^{\circ}, \mathrm{Kp}$, Ea e ao cálculo de quantidade de substância em uma reação química? Quais níveis de desenvolvimento da habilidade de interpretar a reação química como sistema são características das respostas dos candidatos ao curso de licenciatura em Química?

\section{Pressupostos e aspectos metodológicos}

A pesquisa apresentada assume um caráter que relaciona, de forma dialética, as dimensões qualitativa e quantitativa. Em relação à dimensão qualitativa, fez-se uma análise das respostas dos estudantes com a intenção de, ao comparar com as expectativas de respostas definida pela banca examinadora, compreender o sentido atribuído por eles acerca da reação química como um sistema. A dimensão quantitativa se refere aos dados numéricos que permitem caracterizar a sua extensão.

Consideramos que as partes estruturais da pesquisa constituem um sistema, no qual se estabelecem relações substantivas e diversas. Nesse sistema, a escolha dos métodos e dos instrumentos e a definição da metodologia dependem tanto da natureza e dos objetivos da pesquisa como também da matriz teórica que determinam, por sua vez, a perspectiva de análise e a interpretação dos dados.

O estudo foi feito a partir da resposta dada por 126 candidatos que concorriam às vagas destinadas ao curso de Licenciatura em Química, da Universidade Federal do Rio Grande do Norte (UFRN), no Vestibular 2013. Segundo Beltrán Núñez e Ramalho (2012), o vestibular da UFRN tinha como principal função selecionar estudantes para o início dos estudos na educação superior. Esse processo avaliava um conjunto de habilidades e competências que integravam o conteúdo das disciplinas do Ensino Médio.

A questão objeto do estudo, apresentada na Figura 1, é de natureza discursiva com três itens. Nela, é apresentada uma situação-problema, exigindo a interpretação de parâmetros que caracterizam uma reação química (itens a e c), assim como a integração desses parâmetros na aplicação da reação química como um sistema, em uma totalidade (item b).

As provas utilizadas como fonte de informação foram disponibilizadas pelo Observatório da Vida do Estudante Universitário (OVEU) e cedidas pela Comissão Permanente de Vestibular (COMPERVE) da UFRN. A análise das respostas dos candidatos possibilitou determinar os níveis da habilidade em estudo, identificar e caracterizar os erros assim como inferir dificuldades de aprendizagem que podem a eles serem associadas. 
Figura 1. Questão objeto de Estudo

\begin{abstract}
Questão 5
Na história da Química, um dos estudos mais significativos foi desenvolvido pelo químico alemão Fritz Haber para a obtenção da amônia $\left(\mathrm{NH}_{3}\right)$. Essa substância é estrategicamente importante para outros processos da indústria química assim como para a produção de fertilizantes. $O$ processo foi aperfeiçoado posteriormente por Carl Bosch. A importância dessas pesquisas valeu a Haber, em 1918, e a Bosch, em 1931, o Prêmio Nobel de Química.

$O$ processo de obtenção da amônia na indústria se realiza a partir da reação do $H_{2(g)} \operatorname{com} \circ \mathrm{N}_{2(\mathrm{~g})}$, mostrada através da equação a seguir:

$3 \mathrm{H}_{2(\mathrm{~g})}+\mathrm{N}_{2(\mathrm{~g})} \rightleftarrows 2 \mathrm{NH}_{3(\mathrm{~g})} ; \Delta \mathrm{G}^{\circ}=-16,45 \mathrm{KJ} / \mathrm{mol} ; \Delta \mathrm{H}^{\circ}<<1 ; \mathrm{Kp}<1 ; \mathrm{P}=1 \mathrm{~atm} ; \mathrm{T}=298,15 \mathrm{~K}$

Para que esse processo ocorra e seja economicamente viável, os pesquisadores mostraram que deve acontecer a altas temperaturas, a alta pressão de forma tal a compensar o efeito da temperatura, e na presença de um catalisador por ter alto valor a energia de ativação da etapa lenta do mecanismo da reação, se comparada com outras reações nessas condições. Considerando essa situação,

A) qual o significado de $\Delta G^{\circ}=-16,45 \mathrm{KJ} / \mathrm{mol}$, de $\mathrm{Kp}<1$ e do valor da energia de ativação observado na etapa informada?

B) por que, para compreender se uma reação química acontece na prática, é importante conhecer os valores dos parâmetros $\Delta \mathrm{G}^{\circ}$, de Kp e o da energia de ativação?

C) para produzir diariamente oito toneladas de amônia, qual a quantidade necessária de hidrogênio, considerando-se que se dispõe de quantidade suficiente de nitrogênio?
\end{abstract}

Fonte: Comperve (2013).

\title{
Processo de análise das respostas da questão da prova
}

Para responder à primeira pergunta de estudo, ou seja, quais erros e dificuldades são característicos de conhecimento dos estudantes sobre reações químicas como um sistema, foram analisadas as respostas para o item a da prova, no qual se avaliava o conhecimento dos parâmetros $\Delta \mathrm{G}^{\mathrm{o}}, \Delta \mathrm{H}^{\mathrm{o}}$ e a Energia de Ativação da reação nas condições dadas. Essas respostas foram categorizadas em Correta, Parcialmente Correta e Incorreta, o que possibilitou identificar, para cada parâmetro, os erros conceituais, os quais, por sua vez, foram relacionados com possíveis dificuldades de aprendizagem, como hipóteses explicativas selecionadas na revisão da literatura.

São identificados como erros os desvios das respostas considerando-se o conhecimento da Química em relação aos significados dos parâmetros, como explicam Beltrán Núñez e Ramalho (2012). Já a dificuldade de aprendizagem é, hipoteticamente, o que explica o erro. Dessa forma, o erro constitui uma manifestação fenomenológica da dificuldade de aprendizagem, enquanto essência.

Para responder à segunda questão de estudo, foram analisadas as respostas de todos os itens na sua integralidade, a fim de que se pudesse determinar os níveis de desenvolvimento que fazem parte da habilidade de explicar uma reação química, nesse caso, a síntese do $\mathrm{NH}_{3(\mathrm{~g})}$ como um sistema complexo, segundo a perspectiva teórica assumida na pesquisa.

Para essa finalidade, foi estabelecido um nível teórico (nível 1) que corresponde à resposta correta desejada, conforme explicitado no Quadro 1. 
Quadro 1. Nível 1 de desenvolvimento da habilidade interpretar a reação química como sistema complexo

\begin{tabular}{|c|l|}
\hline Descrição & \multicolumn{1}{c|}{ Nível de desenvolvimento } \\
\hline Item a & $\begin{array}{l}\text { Explicita corretamente o significado de } \Delta \mathrm{G}^{\circ} \text { (negativo), de } \mathrm{Kp}<1 \text { e o da energia de } \\
\text { ativação elevada para a reação química. }\end{array}$ \\
\hline Item b & $\begin{array}{l}\text { Apresenta, na resposta, a integração de forma sistêmica dos significados de } \Delta \mathrm{G}^{\circ} \text {, de } \\
\text { Kp e energia de ativação para a ocorrência da reação química. }\end{array}$ \\
\hline Item c & Calcula corretamente a massa de hidrogênio. \\
\hline
\end{tabular}

Fonte: elaborado pelos autores.

Como se observa no quadro, esse nível supõe a capacidade de integrar as dimensões qualitativa/quantitativa da reação química (item c), com as dimensões termodinâmica do equilíbrio e da cinética (item a), assim como explicar a razão pela qual se necessita dos três parâmetros para evidenciar a ocorrência da reação química nas condições dadas. As análises das respostas à questão possibilitaram estabelecer níveis diferentes, de forma empírica.

Os dados das análises relacionados às duas perguntas da pesquisa foram organizados em uma folha de registro, permitindo o tratamento em uma planilha do EXCEL.

\section{O conhecimento das reações químicas como sistema complexo}

Os resultados da pesquisa e suas análises são apresentados segundo as questões de estudo formuladas, de modo tal a atingir o objetivo geral, ou seja, caracterizar o conhecimento dos candidatos sobre a reação química como um sistema complexo.

No item a, interessava apresentar e discutir os resultados relacionados com a compreensão da Energia Livre de Gibbs e sua variação $\left(\Delta \mathrm{G}^{\circ}\right)$ como parâmetro termodinâmico, que é imprescindível para que se compreenda a reação química como sistema complexo ao expressar a espontaneidade do processo.

Tabela 1. Proporção de estudantes segundo o tipo de respostas para a interpretação da energia de Gibbs $\left(\Delta \mathrm{G}^{\circ}\right)$

\begin{tabular}{lcc}
\hline \multicolumn{1}{c}{ Tipos de Resposta } & Quantidade & Porcentagem \\
\hline Correta & 12 & $9,5 \%$ \\
Parcialmente correta & 3 & $2,4 \%$ \\
Incorreta & 73 & $57,9 \%$ \\
Em branco & 38 & $30,2 \%$ \\
Total & $\mathbf{1 2 6}$ & $\mathbf{1 0 0 , 0} \%$ \\
\hline
\end{tabular}

Fonte: elaborada pelos autores.

Nessa tabela, constata-se que a maioria dos estudantes respondeu de forma incorreta $(57,9 \%)$ ou deixaram de responder $(30,2 \%)$, ou seja, esses estudantes não expressam domínio do sentido do parâmetro $\left(\Delta \mathrm{G}^{\circ}\right)$, relevante na compreensão da reação química. 
Para o caso das respostas parcialmente corretas e incorretas, ou seja, a não associação correta do valor com a espontaneidade da reação, foi feita a análise dos erros. A Tabela 2 apresenta os erros mais recorrentes dos estudantes na interpretação do parâmetro da variação da Energia Livre de Gibbs $\left(\Delta G^{\circ}\right)$.

Tabela 2. Erros recorrentes na definição do conceito de Variação da Energia Livre de Gibbs $\left(\Delta \mathrm{G}^{\circ}\right)$

\begin{tabular}{lcc}
\hline \multicolumn{1}{c}{ Erro em relação à definição do parâmetro $\Delta \mathbf{G}^{\circ}$} & Quantidade & Porcentagem \\
\hline Considerar $\Delta \mathrm{G}^{\circ}$ como $(\Delta \mathrm{H})$ & 15 & $19,7 \%$ \\
Confundir $\Delta \mathrm{G}^{\circ}$ com temperatura da reação & 10 & $13,2 \%$ \\
Atribuir ao $\Delta \mathrm{G}^{\circ}$ o sentido da temperatura de ebulição & 9 & $11,9 \%$ \\
Confundir $\Delta \mathrm{G}^{\circ}$ com Entropia da reação & 6 & $7,9 \%$ \\
Confundir a definição do conceito de espontaneidade & 10 & $13,2 \%$ \\
Atribuir ao $\Delta \mathrm{G}^{\circ}$ um sentido cinético & 38 & $50 \%$ \\
\hline
\end{tabular}

Fonte: elaborada pelos autores.

A análise da tabela permite constatar que os estudantes atribuem ao parâmetro termodinâmico um sentido cinético (50\%). Outra quantidade de estudantes $(19,7 \%)$ não diferencia $\Delta G^{\circ}$ como critério de espontaneidade, mas como liberação de energia (reação exotérmica). Várias pesquisas, dentre elas as de Quilez (2009), têm mostrado dificuldades de aprendizagem dos estudantes em relação à interpretação do parâmetro termodinâmico Energia Livre de Gibbs. É importante ressaltar que os percentuais totais apresentados estão acima dos 100\% porque houve estudantes que cometeram mais de um erro ao responder o item.

Estudos Diante dessa titui como Química,s tema?

er sobre o equilíbrio químico possibilitam analisar as condições que favorecem a extensão ou não de uma dada reação química em equilíbrio, partindo do pressuposto de que, teoricamente, toda reação é de equilíbrio. No caso da síntese da amônia $\left(\mathrm{NH}_{3(\mathrm{~g})}\right)$, o Kp $<1$ a T. $\mathrm{Na}$ Tabela 3, apresentam-se os índices relativos aos tipos de resposta dada pelos estudantes quanto à interpretação da Constante de Equilíbrio (Kp).

Tabela 3. Proporção de estudantes segundo o tipo de resposta na interpretação da Constante de Equilíbrio (Kp)

\begin{tabular}{lcc}
\hline \multicolumn{1}{c}{ Tipo de resposta } & Quantidade & Porcentagem \\
\hline Correta & 1 & $0,8 \%$ \\
Parcialmente correta & 6 & $4,8 \%$ \\
Incorreta & 69 & $54,7 \%$ \\
Em branco & 50 & $39,7 \%$ \\
Total & $\mathbf{1 2 6}$ & $\mathbf{1 0 0 , 0} \%$ \\
\hline
\end{tabular}

Fonte: elaborada pelos autores a partir dos dados da pesquisa. 
Nessa tabela, verifica-se que a maioria dos estudantes (54,7\%) interpretou de forma incorreta o valor de Kp da reação apresentada, enquanto 39,7\% , não responderam, evidenciando dificuldades referentes ao conhecimento do significado desse outro parâmetro, que caracteriza uma reação química.

Das análises das respostas dos estudantes, foi possível identificar os erros mais relevantes em relação à interpretação do Kp. A Tabela 4 apresenta os erros mais recorrentes na interpretação do parâmetro da constante de equilíbrio (Kp).

Tabela 4. Erros recorrentes na definição do conceito de constante de equilíbrio (Kp)

\begin{tabular}{lcc}
\hline \multicolumn{1}{c}{ Erro em relação ao sentido do parâmetro (kp) } & Quantidade & Porcentagem \\
\hline Confundir Kp com Kps & 16 & $36,5 \%$ \\
Atribuir a Kp o sentido da Kc & 19 & $25,3 \%$ \\
Atribuir a Kp critérios de espontaneidade da reação & 3 & $4,0 \%$ \\
Atribuir a Kp sentido de $\Delta \mathrm{H}$ & 2 & $2,7 \%$ \\
Confundir Kp com Energia de Ativação (Ea) & 3 & $4,0 \%$ \\
Somente representaram a expressão do equilíbrio em termos de & 16 & $36,5 \%$ \\
pressão & 13 & $17,3 \%$ \\
Atribuir a Kp o conceito de energia de ativação & 13 & $17,3 \%$ \\
Confundir Kp com pressão do sistema & & \\
\hline
\end{tabular}

Fonte: elaborada pelos autores.

Semelhante a nossos resultados, Raviolo e Martínez Aznar (2003) constataram erros dos estudantes ao confundir Kp com a energia de ativação. Nesse mesmo sentido, Caamaño (2003) também encontrou esse tipo erro.

Mortimer e Miranda (1995) afirmaram que autores alertam para o fato de o ensino de química privilegiar o uso de equações para a representação de reações químicas, ficando, em segundo plano, o estudo dos fenômenos envolvidos nas transformações químicas. Essa ênfase pode influenciar na manutenção de conceitos diferentes dos científicos, por parte dos alunos que apresentam dificuldades em relacionar as transformações que ocorrem em nível fenomenológico com as explicações no nível atômico-molecular.

Em relação às dificuldades de aprendizagem de estudantes sobre o sentido do valor da Kp, autores como Martínez-Grau, Solaz-Portolés e Sanjosé (2014) chamam a atenção para os conteúdos desse parâmetro, nos livros didáticos, serem uma das fontes de dificuldades de aprendizagem. No mesmo sentido, Raviolo e Martínez Aznar (2003) têm ressaltado dificuldades relacionadas à própria natureza desse conteúdo e a suas representações.

$\mathrm{Na}$ Tabela 5 apresenta-se o desempenho dos estudantes em relação à interpretação da Energia de Ativação (EA), um parâmetro relevante na compreensão da reação química como um sistema complexo. Enquanto os parâmetros $\Delta \mathrm{H}^{0}$ se referem aos processos energéticos, o $\Delta \mathrm{G}^{\circ}$, à espontaneidade e o Kp, à extensão da reação, os parâmetros cinéticos dizem respeito à velocidade das reações nas condições dadas. 
Tabela 5. Porcentagem de erros na definição do conceito de energia de ativação (EA)

\begin{tabular}{lcc}
\hline \multicolumn{1}{c}{ Tipo de resposta } & Quantidade & Porcentagem \\
\hline Correta & 13 & $10,3 \%$ \\
Parcialmente correta & 12 & $9,5 \%$ \\
Incorreta & 27 & $21,4 \%$ \\
Em branco & 74 & $58,8 \%$ \\
Total & $\mathbf{1 2 6}$ & $\mathbf{1 0 0 , 0} \%$ \\
\hline
\end{tabular}

Fonte: elaborada pelos autores.

Os resultados apresentados na Tabela 5 são reveladores, pois apresentam as dificuldades dos estudantes para interpretar, de forma correta, o valor da energia de ativação em um processo químico, como no caso da síntese da amônia. Constata-se que a maioria dos estudantes respondeu de forma incorreta $(21,4 \%)$ ou deixou de responder (58,8\%). Apenas 10,3\% dos estudantes responderam de forma correta ao atribuir um elevado valor à EA na etapa lenta do processo, o que exige a presença de um catalizador para aumentar a velocidade da reação.

Os erros dos estudantes quanto ao sentido da Energia de Ativação são apresentados na Tabela 6.

Tabela 6. Erros recorrentes na definição da Energia de Ativação

\begin{tabular}{lcc}
\hline Erro em relação a definição da Energia de Ativação (EA) & Quantidade & Porcentagem \\
\hline Atribuir à EA o sentido de velocidade da reação & 8 & $20,5 \%$ \\
Atribuir a EA a variação da Entalpia $(\Delta \mathrm{H})$ da reação & 10 & $25,7 \%$ \\
Confundir EA e Catalizador de uma reação & 7 & $17,9 \%$ \\
Considerar a EA como o Complexo Ativado & 3 & $7,7 \%$ \\
Confundir EA e a constante de equilíbrio (Kp) & 5 & $12,8 \%$ \\
Confundir EA com a temperatura $(\mathrm{T})$ da reação & 6 & $15,4 \%$ \\
\hline
\end{tabular}

Fonte: elaborada pelos autores.

Nessa tabela, é possível constatar que a maioria dos estudantes $(80,2 \%)$ não evidencia conhecer o sentido do valor da EA em uma reação química, questão estudada por Caamaño (1994).

Para determinar os níveis de desenvolvimento da habilidade de interpretar a reação química como um sistema complexo, foram analisadas e comparadas as respostas dos estudantes com as esperadas para o nível 1, definidas teoricamente, o que permitiu estabelecer, de forma empírica, 12 níveis diferentes, apresentados no Quadro 2. 
Quadro 2. Níveis de desenvolvimento da habilidade de interpretar a reação química como sistema complexo

\begin{tabular}{|c|c|c|c|}
\hline Nível & Características & Quantidade & Porcentagem \\
\hline 1 & $\begin{array}{l}\text { Item a - o estudante responde corretamente o conceito } \\
\text { de } \Delta \mathrm{G}^{\circ}, \mathrm{Kp} \text { e energia de ativação (EA). } \\
\text { Item b - apresenta, de forma sistêmica, a integração dos } \\
\text { significados de } \Delta \mathrm{G}^{\circ} \text {, de Kp e de energia de ativação (EA) } \\
\text { para compreensão da reação química. } \\
\text { Item c - calcula corretamente a massa de hidrogênio. }\end{array}$ & 0,0 & 0,0 \\
\hline 2 & $\begin{array}{l}\text { Item a - o estudante responde corretamente um dos sig- } \\
\text { nificados: } \Delta \mathrm{G}^{\circ}, \mathrm{Kp} \text { ou energia de ativação (EA). } \\
\text { Item b - apresenta, de forma sistêmica, a integração, de } \\
\text { dois significados: } \Delta \mathrm{G}^{\circ}, \mathrm{Kp} \text { ou energia de ativação (EA) } \\
\text { para a compreensão da reação química. } \\
\text { Item c - calcula corretamente a massa de hidrogênio }\end{array}$ & 2 & 1,6 \\
\hline 3 & $\begin{array}{l}\text { Item a - o estudante responde corretamente dois dos } \\
\text { significados: } \Delta \mathrm{G}^{\circ}, \mathrm{Kp} \text { ou energia de ativação (EA). } \\
\text { Item b - não apresenta, de forma sistêmica, a correta } \\
\text { integração dos significados de } \Delta \mathrm{G}^{\circ} \text {, de Kp e de energia de } \\
\text { ativação (EA) para a ocorrência da reação química. } \\
\text { Item c - calcula corretamente a massa de hidrogênio }\end{array}$ & 2 & 1,6 \\
\hline 4 & $\begin{array}{l}\text { Item a - o estudante responde corretamente um dos sig- } \\
\text { nificados: } \Delta \mathrm{G}^{\circ}, \mathrm{Kp} \text { ou energia de ativação }(\mathrm{EA}) \text {. } \\
\text { Item b - não apresenta, de forma sistêmica, a correta } \\
\text { integração, dos significados de } \Delta \mathrm{G}^{\circ} \text {, de Kp e de energia } \\
\text { de ativação (EA) para a ocorrência da reação química. } \\
\text { Item c - calcula corretamente a massa de hidrogênio. }\end{array}$ & 3 & 2,4 \\
\hline 5 & $\begin{array}{l}\text { Item a - o estudante responde corretamente um dos sig- } \\
\text { nificados: } \Delta G^{\circ}, \mathrm{Kp} \text { ou energia de ativação (EA). } \\
\text { Item b - não apresenta, de forma sistêmica, a correta } \\
\text { integração dos significados de } \Delta \mathrm{G}^{\circ} \text {, de Kp e de energia de } \\
\text { ativação (EA) para a ocorrência da reação química. } \\
\text { Item c - calcula incorretamente a massa de hidrogênio. }\end{array}$ & 14 & 11,1 \\
\hline 6 & $\begin{array}{l}\text { Item a - o estudante não responde corretamente nenhum } \\
\text { dos significados: } \Delta G^{\circ}, \mathrm{Kp} \text { ou energia de ativação (EA). } \\
\text { Item b - não apresenta, de forma sistêmica, a integração } \\
\text { dos significados de } \Delta \mathrm{G}^{\circ} \text {, de Kp e de energia de ativação } \\
\text { para a ocorrência da reação química. } \\
\text { Item c - calcula corretamente a massa de hidrogênio }\end{array}$ & 2 & 1,6 \\
\hline 7 & $\begin{array}{l}\text { Item a - o estudante não responde corretamente nenhum } \\
\text { dos significados: } \Delta \mathrm{G}^{\circ}, \mathrm{Kp} \text { ou energia de ativação (EA). } \\
\text { Item b - não apresenta, de forma sistêmica, a integração } \\
\text { dos significados de } \Delta \mathrm{G}^{\circ} \text {, de Kp e de energia de ativação } \\
\text { para a ocorrência da reação química. } \\
\text { Item c - calcula incorretamente a massa de hidrogênio. }\end{array}$ & 46 & 36,5 \\
\hline
\end{tabular}


Erros e dificuldades de aprendizagem de estudantes ...

Quadro 2. continuação

\begin{tabular}{|c|c|c|c|}
\hline Nível & Características & Quantidade & Porcentagem \\
\hline 8 & $\begin{array}{l}\text { Item a - o estudante não responde corretamente nenhum } \\
\text { dos significados: } \Delta G^{\circ}, K p \text { ou energia de ativação (EA). } \\
\text { Item b - apresenta, de forma sistêmica, a integração de } \\
\text { dois significados: } \Delta G^{\circ}, K p \text { ou energia de ativação (EA) } \\
\text { para a compreensão da reação química. } \\
\text { Item c - deixa em branco. }\end{array}$ & 1 & 0,8 \\
\hline 9 & $\begin{array}{l}\text { Item a - deixa em branco. } \\
\text { Item b - apresenta, de forma sistêmica, a integração de } \\
\text { dois significados: } \Delta G^{\circ}, \text { Kp ou energia de ativação (EA) } \\
\text { para a compreensão da reação química. } \\
\text { Item c - calcula incorretamente a massa de hidrogênio. }\end{array}$ & 11 & 8,7 \\
\hline 10 & $\begin{array}{l}\text { Item a - o estudante não responde corretamente nenhum } \\
\text { dos significados: } \Delta \mathrm{G}^{\circ}, \mathrm{Kp} \text { ou energia de ativação (EA). } \\
\text { Item b - deixa em branco. } \\
\text { Item } \mathrm{c} \text { - deixa em branco. }\end{array}$ & 8 & 6,3 \\
\hline 11 & $\begin{array}{l}\text { Item a - deixa em branco. } \\
\text { Item } \mathrm{b} \text { - deixa em branco. } \\
\text { Item } \mathrm{c} \text { - calcula corretamente a massa de hidrogênio. }\end{array}$ & 1 & 0,8 \\
\hline 12 & $\begin{array}{l}\text { Item } \mathrm{a} \text { - deixa em branco. } \\
\text { Item } \mathrm{b} \text { - deixa em branco. } \\
\text { Item } \mathrm{c} \text { - deixa em branco. }\end{array}$ & 36 & 28,6 \\
\hline
\end{tabular}

Fonte: elaborado pelos autores.

No Quadro 2 constata-se que, para os níveis 2, 3 e 4, os estudantes caracterizam, de forma correta, um ou dois parâmetros, mas não integram, de forma adequada, os três parâmetros, o que evidenciaria a compreensão da reação química como sistema, além de não calcular corretamente a quantidade de $\mathrm{H}_{2}(\mathrm{~g})$.

Para o nível 5, 11,1\% dos estudantes apenas respondem corretamente o significado de um dos parâmetros da reação, consequentemente, não conseguem fazer a integração com os outros parâmetros e fazem o cálculo da massa de hidrogênio de forma incorreta.

Além disso, a maioria dos estudantes se situam nos níveis 7 (36,5\% não respondem corretamente a nenhum dos itens), 9 (8,7\% dos estudantes só articulam dois parâmetros, calculam incorretamente a massa do hidrogênio) ou não respondem a questão (28,6\% dos candidatos).

Outra constatação feita a partir desse quadro é que nenhum dos estudantes mostra um conhecimento adequado da reação química como um sistema complexo. Apenas 12 estudantes $(9,5 \%)$ se referem à necessidade de integrar apenas dois dos parâmetros, o que evidência uma visão limitada do sistema.

Considera-se que a questão é de elevado nível de dificuldade para os estudantes, uma vez que nenhum conseguiu relacionar os três parâmetros $\Delta \mathrm{G}^{\circ}, \mathrm{Kp}$ e EA. Ela demanda um conhecimento sistêmico do tema, no qual os parâmetros $\Delta \mathrm{G}^{\circ}$, Kp e EA sejam relacionados às 
condições de espontaneidade, rendimento e energia para as ocorrências das reações químicas nas condições dadas no problema.

Uma pesquisa sobre Química Geral de Beltrán Núñez (1994), realizada com estudantes em um curso universitário, revelou que somente aqueles que participaram de um processo de formação da habilidade de interpretar reações químicas como um sistema complexo, sob a orientação teórica de Reshetova (1988), atingiram resultados positivos, evidenciando o domínio da habilidade na qual se integram conceitos e modelos teóricos correspondentes a esse tipo de sistema químico.

Esses resultados evidenciaram, por sua vez, que o pensamento sistêmico dialético não se formava de forma espontânea, mas como resultado de um processo planejado para esse propósito.

O pensamento sistêmico dialético, como têm explicado Reshetova (1988) e Afanasiev (1979), supõe integrar as partes em uma totalidade que apresenta novas características em relação a estas e revelar, no sistema, as contradições que articulam as dimensões funcionais e estruturais da habilidade. Dessa forma, exige um percurso da aprendizagem do geral para o singular, do abstrato para o concreto e vice-versa, com um enfoque intradisciplinar (BELTRÁN NÚÑEZ, 2009).

\section{Conclusão}

A compreensão da reação química em sua totalidade, ou seja, como um sistema complexo, é uma condição essencial para o estudo da Química no ensino médio, o que supõe superar a visão fragmentada dos conteúdos e uma postura mais intradisciplinar que revele as relações entre diferentes temas e conteúdos da Química.

Este estudo, realizado com as respostas dos estudantes em relação à interpretação da reação de síntese da amônia como um sistema complexo, revelou um conjunto de erros e dificuldades de aprendizagem de grande importância para se repensar o ensino dos conteúdos das reações químicas no Ensino Médio.

Foi possível constatar que os estudantes apresentam erros e dificuldades de aprendizagens ao interpretarem os parâmetros que dizem respeito à compreensão adequada de $\Delta \mathrm{H}^{\circ}$, $\Delta \mathrm{G}^{\mathrm{o}}$ e Energia de Ativação em uma reação química, o que tem sido também discutido em vários artigos. Por sua vez, revelou também um baixo desempenho dos estudantes na interpretação da reação química como um sistema, o que exigia a integração desses parâmetros que caracterizam uma reação química.

Ao analisar as dificuldades de aprendizagem como possíveis causas dos erros (BELTRÁN NÚÑEZ; RAMALHO, 2012), deve-se destacar as contribuições de Kempa (1991) demonstrando que muitas delas podem estar relacionadas à natureza das ideias prévias dos estudantes, à incapacidade para estabelecer relações entre os conceitos e para organizar e processar informações assim como a aspectos de natureza linguística. Por sua vez, como explicam Galagovsky, Di Giacomo e Alí (2015), essas dificuldades podem ser decorrentes da confusão no uso de vários modelos para um mesmo conteúdo.

Interpretar uma reação, como no caso da síntese da amônia $\left(\mathrm{NH}_{3(\mathrm{~g})}\right)$, exige elevada capacidade de abstração, pois isso requer a aplicação de diversos modelos científicos, o uso 
adequado da linguagem da Química e o estabelecimento de relações complexas entre dimensões estudadas como aparentemente desconexas, o que se constitui como fonte de erros na aprendizagem. Diante dessa situação, a pesquisa traz contribuições significativas, uma vez que identifica erros e dificuldades de aprendizagem referentes à compreensão da reação Química como um sistema complexo, ou seja, não só à compreensão das dimensões da reação (qualitativa, quantitativa, energética, dinâmica e de extensão) como, tradicionalmente, é ensinado e pesquisado, mas à integração desses aspectos. Dessa forma, o trabalho aporta resultados que podem contribuir para que sejam propostas novas estratégias de ensino relativas às reações químicas como sistemas complexos e para o desenvolvimento do pensamento teórico.

\section{Referências}

AFANASIEV, V. El enfoque sistémico aplicado al conocimiento social. Revista de Ciencias Sociales, Moscú, v. 35, n. 1, 1979.

BELTRÁN NÚÑEZ, I. Sistema didáctico para la enseñanza de la química general. 1994. Tesis (Doctorado en Ciencias Pedagógicas) - Universidad de la Habana, La Habana, 1994. Liber Livro, 2009.

Vygotsky, Leontiev, Galperin: formação de conceitos princípios didáticos. Brasília: BELTRÁN NÚÑEZ, I.; RAMALHO, B. L. (Org.). Estudos de erros e dificuldades de aprendizagem: as provas de química e biologia do vestibular da UFRN. Natal: EDUFRN, 2012.

BELTRÁN NÚÑEZ, I.; UEHARA, F. M. G. Análise pedagógica das provas de química do vestibular 2008. In: BELTRÁN NÚÑEZ, I.; RAMALHO, B. L. (Org). Estudos de erros e dificuldades de aprendizagem: as provas de química e biologia do vestibular da UFRN. Natal: EDUFRN, 2012. p. 37-79.

BRASIL. Ministério da Educação. Diretrizes curriculares nacionais do ensino médio. Brasilia, 2013.

CAAMAÑO, A. Concepcions dels alumnes sobre la composició i estructura de la matèria i sobre el canvi químic: comprensió de les formes simbòliques de representació. 1994. Tesis (Doctoral - Didáctica de la Química) - Facultad de Químicas, Universidad de Barcelona, Barcelona, 1994.

La enseñanza y el aprendizaje de la química. In: JIMÉNEZ ALEIXANDRE, M. P. et al. (Coord.). Enseñar ciencias. 2. ed. Barcelona: Graó, 2007. p. 95- 118.

. Los trabajos prácticos en ciencias. In: JIMÉNEZ ALEIXANDRE, M. P. et al.

(Coord.). Enseñar ciencias. 2. ed. Barcelona: Graó, 2007. p. 95-118. 
CARBONELL, F; FURIÓ-MAS, C. Opiniones de los adolescentes respecto al cambio sustancial de las reacciones químicas. Enseñanza de las Ciencias, Barcelona, v. 5, n. 1, p. 3-9, 1987. Disponível em: <http://www.raco.cat/index.php/Ensenanza/article/ view/50911/92811>. Acesso em: 30 jan. 2018.

COMPERVE. Exame vestibular 2013: prova discursiva de química, questão 5. Natal: UFRN, 2013.

FURIÓ, C.; FURIÓ, C. Dificultades conceptuales y epistemológicas en el aprendizaje de los procesos químicos. Educación Química, Buenos Aires, v. 11, n. 3, p. 300-308, 2000. Disponível em: <https://rodas5.us.es/file/9ea0c662-b500-306c-5a5a-942a4a004642/2/ texto3_SCORM.zip/index.html>.Acesso em: 30 jan. 2018.

GALAGOVSKY, L. R.; DI GIACOMO, M. A.; ALÍ, S. Estequiometria y ley de conservación de la massa: lo que puede ocultar la simplificación del discurso esperto.

Ciência \& Educação, Bauru, v. 21, n. 2, p. 351-360, 2015. Disponível em: < https://doi. org/10.1590/1516-731320150020006>. Acesso em: 30 jan. 2018.

JOHNSTONE, A. H. Chemical education research in Glasgow in perspective. Chemistry Education Research and Practice, Cambridge, v. 7, n. 2, p. 49-63, 2006.

. The development of chemistry teaching: a changing response to changing demand. Journal of Chemical Education, Washington, v. 70, n. 9, p. 701-705, 1993.

. Teaching of chemistry: logical or psychological? Chemistry Education: research and practice in Europe, Ioannina, v. 1, n. 1, p. 9-15, 2000.

KEMPA, R. Students learning difficulties in science: causes and possible remedies. Enseñanza de las Ciencias, Barcelona, v. 9, n. 2, p. 119-128, 1991. Disponível em: <http://www.raco.cat/index.php/Ensenanza/article/view/51371/93121>. Acesso em: 30 jan. 2018.

LOPES, A. R. C. Livros didáticos: obstáculos ao aprendizado da ciência química: obstáculos animistas e realistas. Química Nova, São Paulo, v. 15, n. 3, p. 254-261, 1992. Disponível em: <http:/ /quimicanova.sbq.org.br/detalhe_artigo.asp?id=1597>. Acesso em: 30 jan. 2018.

MARTÍNEZ-GRAU, M.; SOLAZ-PORTOLÉS, J. J.; SANJOSÉ, V. Propuesta de un test para determinar el conocimiento conceptual de estudiantes universitarios sobre la constante de equilibrio químico y su aplicación en estudiantes españoles. Química Nova, São Paulo, v. 37, n. 4, p. 740-744, 2014. Disponível em: <https://doi.org/10.5935/01004042.20140118>. Acesso em: 30 jan. 2018.

MENESES, F. M. G. A compreensão de reação química como um sistema complexo a partir da discussão dos erros e dificuldades de aprendizagem de estudantes do ensino médio. 2015. 271 f. Tese (Doutorado em Química) - Universidade Federal do Rio Grande do Norte, Natal, 2015. 
MOL, G. S.; SILVA, R. R. A experimentação no ensino de química como estratégia para a formação de conceito. In: ENCONTRO NACIONAL DE ENSINO DE QUÍMICA, 8., 1996, Campo Grande. Anais... Campo Grande: UFMS, 1996.

MORTIMER, E. F.; MIRANDA, L. C. Transformações: concepções de estudantes sobre reações químicas. Química Nova na Escola, São Paulo, n. 2, p. 23-26, 1995. Disponível em: <http://qnesc.sbq.org.br/online/qnesc02/aluno.pdf>. Acesso em: 30 jan. 2018.

NARDIN C. S.; SALGADO, T. D. M.; DEL PINO, J. C. Análise de uma proposta de ensino de reações químicas entre compostos inorgânicos referenciados em mecanismos de reação. In: V ENCONTRO NACIONAL DE PESQUISA EM EDUCAÇÃO EM CIÊNCIAS, 5., 2005, Bauru. Atas... Disponível em: <http://www.nutes.ufrj.br/abrapec/venpec/conteudo/ artigos/3/pdf/p187.pdf>. Acesso em: 30 jan. 2018.

POZO, J. I.; GÓMEZ C. M. A. A aprendizagem e o ensino de ciências: do conhecimento cotidiano ao conhecimento científico. 5. ed. Porto Alegre: Artmed, 2009.

POZO, J. I. et. al. Las ideas de los alumnos sobre la ciencia: una interpretación desde la psicología cognitiva. Enseñanza de las Ciencias, Barcelona, v. 9, n. 1, p. 83-94, 1991. Disponível em: <http://www.raco.cat/index.php/Ensenanza/article/view/51359/93108>. Acesso em: 30 jan. 2018.

QUILEZ, J. Análisis de los errores que presentan los libros de texto universitários de química general al tratar la energia libre de Gibbs. Enseñanza de las Ciencias, Barcelona, v. 27, n. 3, p. 317-330, 2009. Disponível em: <http://www.raco.cat/index.php/Ensenanza/article/ view/142066/332847>. Acesso em: 30 jan. 2018.

RAVIOLO, A.; MARTÍNEZ AZNAR, M. Una revisión de las concepciones alternativas de los estudiantes en relación con el equilibrio químico: clasificación y síntesis didácticas. Educación Química, Bueno Aires, v. 14, n. 3, p. 60-66, 2003.

RAVIOLO, A. et al. Desarrollo de actitudes hacia el cuidado de la energía: experiencia en la formación de maestros. Enseñanza de las Ciencias, Barcelona, v. 18, n. 1, p. 79-86, 2000. Disponível em: <http://www.raco.cat/index.php/Ensenanza/article/view/21638/21471>. Acesso em: 30 jan. 2018.

RESHETOVA, Z. A. Realización de los principios del enfoque sistêmico em las asignaturas. La Habana: CEPES, 1988.

ROSA, M. I. F. P. S.; SCHNETZLER, R. P. Sobre a importância do conceito de transformação química no processo de aquisição do conhecimento químico. Química Nova na Escola, São Paulo, n. 8, p. 31-35, 1998. Disponível em: < http://www.contagem.mg.gov. $\mathrm{br} /$ arquivos/comunicacao/femcitec_sobreoconceitodatransformacao09.pdf $>$. Acesso em: 30 jan. 2018.

SÁLMINA, N. G. Las actividad cognoscitiva de los alunos y modos de construir la asignatura. La Havana: CEPES, 1988. 
Meneses, F. M. G.; Nuñez, I. B.

TALANQUER, V. Common sense chemistry: a model for understanding students' alternative conceptions. Journal of Chemical Education, Washington, v. 83, n. 5, p. 811-816, 2006.

UEHARA, F. M. G. Refletindo dificuldades de aprendizagem em alunos do ensino médio no estudo do equilíbrio químico. 2005. 100 f. Dissertação (Mestrado em Ensino de Ciências Naturais e Matemática) - Universidade Federal do Rio Grande do Norte, Natal, 2005.

Artigo recebido em 31/10/2016. Aceito em 17/09/2017.

Endereço para contato: Instituto Federal de Educação, Ciência e Tecnologia do Rio Grande do Norte, DIAC, Campus Parnamirim, Rua Antônia de Lima Paiva, 155, Bairro Nova Esperança, Parnamirim, Natal 59015-000, RN, Brasil. 